\title{
Dilated Cardiomyopathy with Eosinophilic Granulomatosis with Polyangiitis in Which Active Myocardial Inflammation Was Only Detected by Endomyocardial Biopsy
}

\author{
Takafumi Nakayama, Shunsuke Murai and Nobuyuki Ohte
}

\begin{abstract}
:
Eosinophilic granulomatosis with polyangiitis (EGPA) is a rare type of systemic vasculitis. Cardiac involvement is the main cause of death in patients with this disease. We herein report a case of congestive heart failure in a patient with EGPA. Neither ${ }^{67} \mathrm{Ga}$ scintigraphy nor cardiac magnetic resonance imaging detected inflammation of the myocardium; however, myocardial biopsy revealed numerous infiltrating inflammatory cells, thereby fulfilling the criteria of inflammatory dilated cardiomyopathy. We improved the left ventricular systolic function by increasing the patient's prednisolone dosage. This case shows that in some cases the detection myocardial inflammation - which allows for appropriate therapy - may only be achieved by myocardial biopsy.
\end{abstract}

Key words: inflammatory dilated cardiomyopathy, eosinophilic granulomatosis with polyangiitis, endocardial biopsy, T lymphocyte, macrophage

(Intern Med 57: 2675-2679, 2018)

(DOI: 10.2169/internalmedicine.0330-17)

\section{Introduction}

Eosinophilic granulomatosis with polyangiitis (EGPA), previously known as Churg-Strauss syndrome, is a rare type of systemic small- and medium-vessel vasculitis that affects multiple organs, including the heart, lungs, nerves, gastrointestinal tract, and skin (1). Although the primary etiology of EGPA remains unclear, infiltrating eosinophils and inflammation appear to affect the target organs, leading to organ dysfunction. Cardiac involvement is the main cause of death in patients with this disease (1), and an effective targeted therapy has not been developed. However, there is growing evidence to support that immunosuppressive therapy can improve dilated cardiomyopathy (DCM) in patients with infiltrating inflammatory cells.

We herein describe the case of a patient with systemic inflammatory disease who had severe left ventricular (LV) systolic dysfunction, which led to decompensated heart failure. We report the methods that were used to detect cardiac inflammation and the therapy that was used in the treatment of this case.

\section{Case Report}

A 69-year-old man with a 10-year history of asthma who had been diagnosed with EGPA 5 years previously presented to Nagoya City University Hospital with dyspnea that had worsened in the past month. A previous physician had treated the patient with antibiotics for one week based on a diagnosis of pneumonia; the patient was referred to our hospital when he did not improve. The man had continued to take prednisolone $(5 \mathrm{mg})$ as maintenance therapy for EGPA.

Blood tests revealed the following findings: leukocyte count $15,400 / \mu \mathrm{L}$, neutrophil count $8,470 / \mu \mathrm{L}$ (55\% leuko-

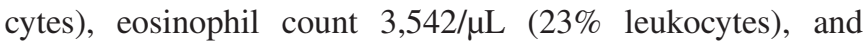
normal lymphocyte and platelet levels. Although his serum levels of C-reactive protein and creatinine kinase were not very elevated $(0.83 \mathrm{mg} / \mathrm{dL}$ and $65 \mathrm{U} / \mathrm{L}$, respectively), the troponin $\mathrm{T}$ level was $0.177 \mathrm{ng} / \mathrm{mL}$. A serum assay for anti- 


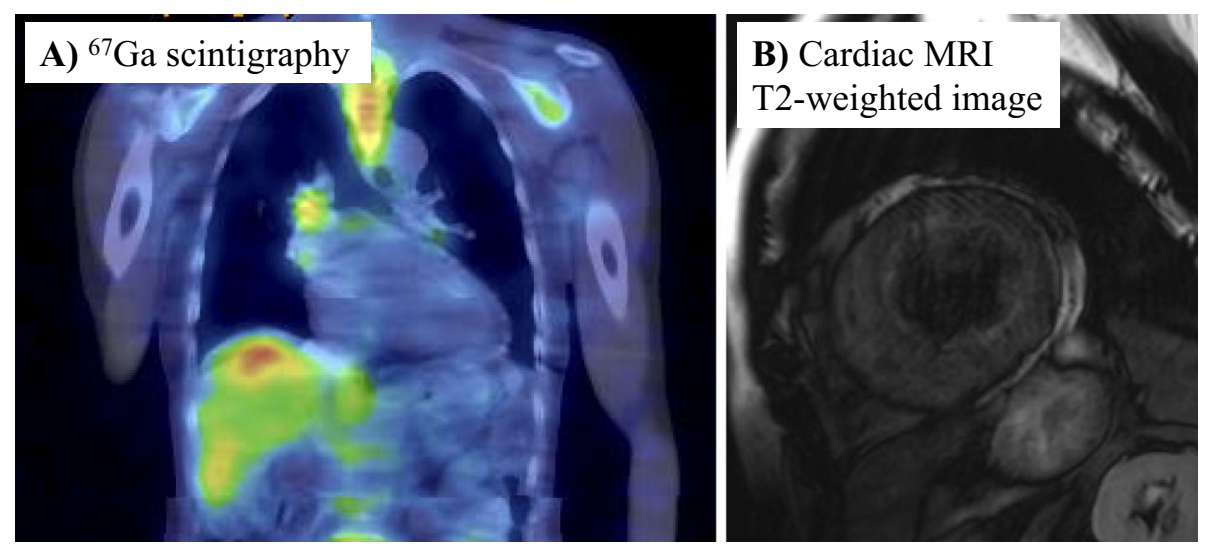

Figure 1. ${ }^{67}$ Ga scintigraphy showed no abnormal uptake by the myocardium (A). Similarly, cardiac magnetic resonance imaging did not show any areas of high intensity in the myocardium (B).
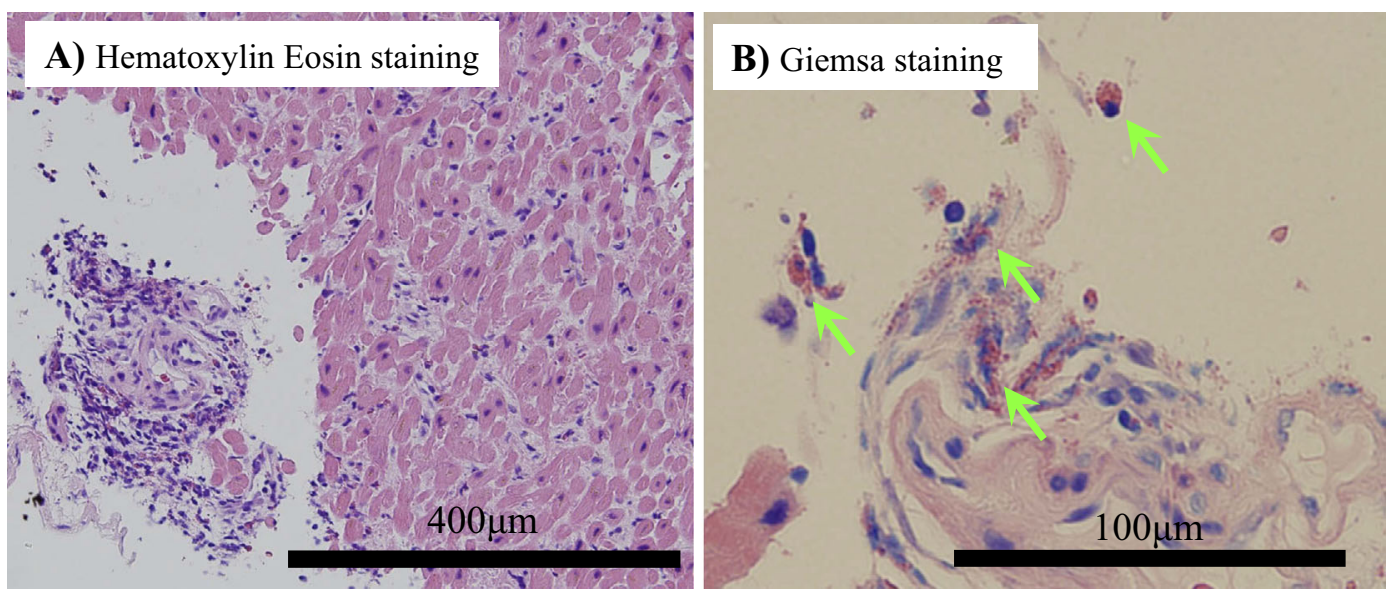

Figure 2. Representative microscopic images of Hematoxylin and Eosin (H\&E) staining (A) and Giemsa-stained tissue samples (B). H\&E staining showed numerous infiltrating inflammatory cells with focal accumulation. Giemsa staining revealed a small number of infiltrating eosinophils.

neutrophil cytoplasmic antibodies was negative.

Chest X-ray revealed pulmonary congestion and an increased cardiothoracic ratio $(66 \%)$ with consolidation, leading to the suspicion of pneumonia. Transthoracic echocardiography showed severe LV systolic dysfunction with no significant valvular disease; the LV end-diastolic and endsystolic diameters were $63 \mathrm{~mm}$ and $53 \mathrm{~mm}$, respectively, and the LV ejection fraction was $29 \%$, indicating a DCMlike heart. We initiated the administration of a loop diuretic and vasodilators based on a diagnosis of acute decompensated left heart failure.

After the heart failure was compensated, we investigated the underlying cause of the LV systolic dysfunction. A diagnosis of coronary artery disease was not supported by coronary angiography, and ${ }^{67} \mathrm{Ga}$ scintigraphy showed no evidence of myocardial inflammation. Cardiac magnetic resonance imaging showed no high intensity regions on T2-weighted imaging (Fig. 1). However, myocardial biopsy specimens from the right ventricular septum showed numerous infiltrating inflammatory cells, including a small number of eosinophils (Fig. 2). The CD3-positive lymphocyte count was 32 cells $/ \mathrm{mm}^{2}$, and the CD68-positive macrophage count was
102 cells $/ \mathrm{mm}^{2}$ (Fig. 3). Based on these findings, we concluded that the patient's LV systolic dysfunction was due to inflammatory dilated cardiomyopathy (DCMi). A myocardial biopsy specimen fulfilled the criteria of DCMi in that the $\mathrm{T}$ lymphocyte and macrophage counts in the myocardium were $\geq 14$ cells $/ \mathrm{mm}^{2}$.

We concluded that the main cause of the worsening LV function was a worsening of EGPA. We therefore decided to increase the dose of oral prednisolone. Because the patient's chest X-ray still showed consolidation that was suspected to represent infectious pneumonia just before the patient's steroid dose was increased, we started prednisolone at a dose of $20 \mathrm{mg} /$ day, which is relatively low for EGPA treatment, based on the suggestion of a pulmonologist. Eosinophils rapidly became undetectable in the patient's blood samples, and the LV systolic function was improved without any $\beta$ blocker treatment. The LV end-diastolic and end-systolic diameters decreased to $48 \mathrm{~mm}$ and $37 \mathrm{~mm}$, respectively, and the LV ejection fraction increased from 29\% to 50\% (Table). Furthermore, as the consolidation on the patient's chest Xray also disappeared within several days, we considered that the consolidation had occurred due to a worsening of EGPA. 

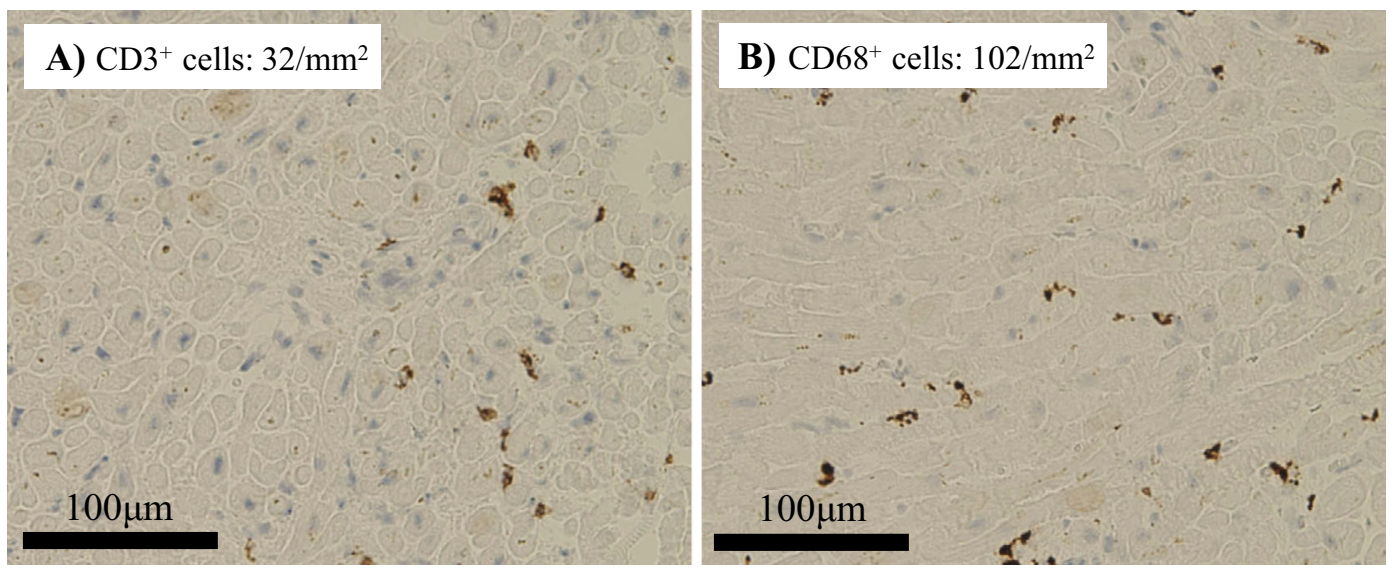

Figure 3. Representative microscopic images showing immunohistochemical staining of biopsy specimens. The signals were specific for CD3 (A) and CD68 (B).

Table. Laboratory and Echocardiographic Data.

\begin{tabular}{lrr}
\hline & $\begin{array}{c}\text { Before increasing } \\
\text { prednisolone }\end{array}$ & $\begin{array}{c}\text { 4 months after increasing } \\
\text { prednisolone }\end{array}$ \\
\hline Laboratory data & & 10.3 \\
WBC, $10^{3} / \mu \mathrm{L}$ & 4.9 & 73 \\
Neut, \% & 55 & 21 \\
Lymph, \% & 16 & 5 \\
Mono, \% & 6 & 0 \\
Eos, \% & 23 & 0 \\
Baso, \% & 0 & 11.7 \\
Hemoglobin, g/dL & 13.1 & 293 \\
Platelet, 10 $3 / \mu \mathrm{L}$ & 194 & 0.4 \\
Total bilirubin, mg/dL & 0.8 & 1.31 \\
Creatinine, mg/dL & 1.14 & 6.1 \\
Total protein, g/dL & 5.7 & 107 \\
BNP, pg/mL & 577 & 0.045 \\
Troponin T, ng/mL & 0.177 & \\
Echocardiographic data & & 48 \\
LVDd, mm & 63 & 37 \\
LVDs, mm & 53 & 9 \\
IVSTd, mm & 8 & 11 \\
PWTd, mm & 8 & 50 \\
LVEF, \% & 29 & 36 \\
LAD, mm & 39 & trivial \\
MR & mild-moderate & \\
\hline
\end{tabular}

WBC: white blood cells, BNP: brain natriuretic peptide, LVDd: left ventricular end-diastolic diameter, LVDs: left ventricular end-systolic diameter, IVSTd: interventricular septal end-diastolic thickness, PWTd: posterior wall end-diastolic thickness, LVEF: left ventricular ejection fraction, LAD: left atrial diameter, MR: mitral regurgitation

The tapering of the prednisolone dose was started after discharge in the outpatient department. The patient was followed to ensure that the LV ejection fraction did not to redeteriorate and that the eosinophil counts in the patient's blood remained below the normal upper limit. At 9 months after the discharge, the patient was taking $7 \mathrm{mg}$ of prednisolone daily without any clinical impairment.

Electrocardiography showed negative $\mathrm{T}$ wave in the precordial and side-wall leads at admission; the finding disap- peared 6 months after increasing the dose of prednisolone. This change might reflect the healing of myocardial inflammation; however, we could not deny the possibility that this electrocardiographic finding was associated with left ventricular volume overload due to acute heart failure (Fig. 4).

\section{Discussion}

The progression of DCM varies according to the patient 

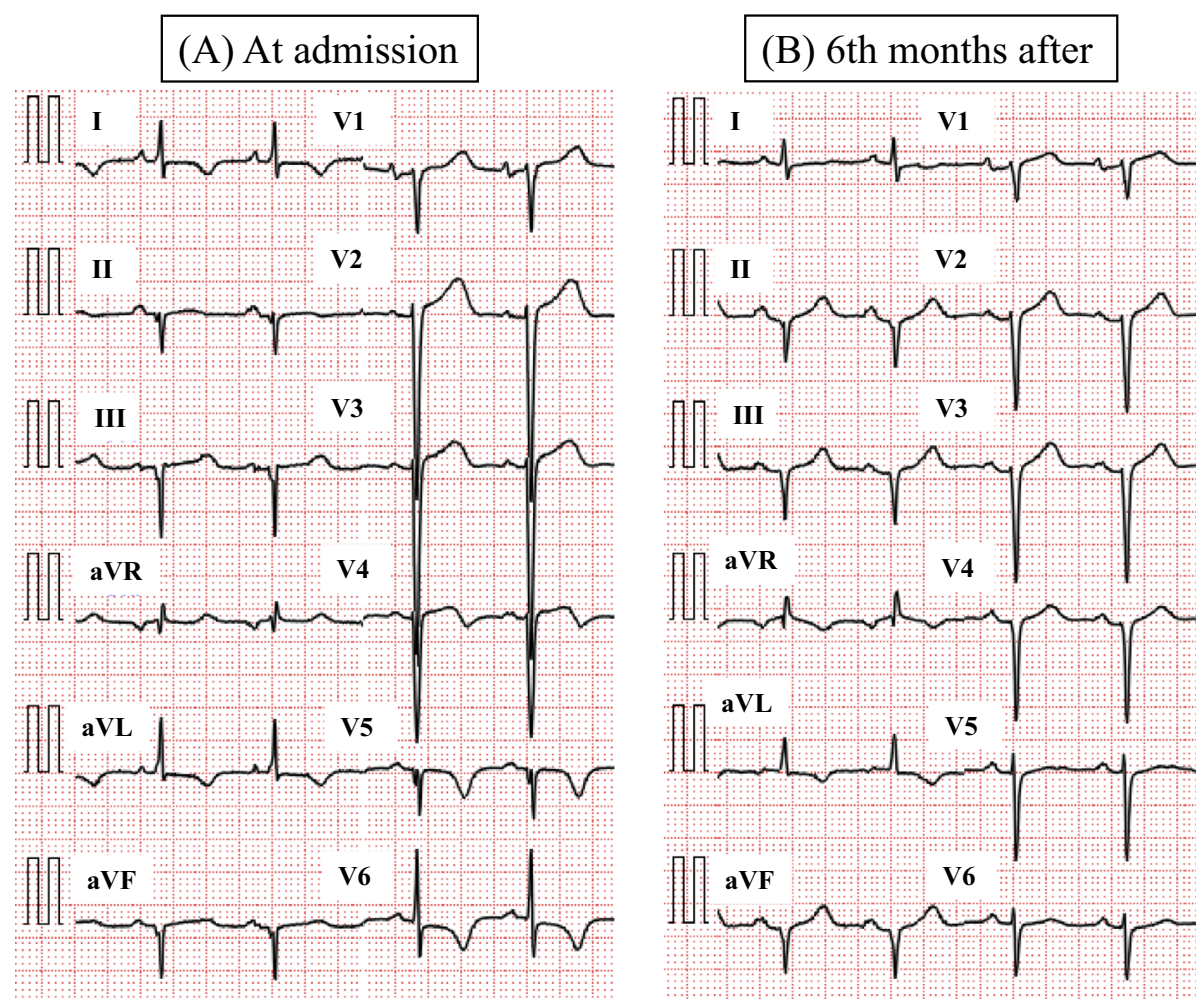

Figure 4. Electrocardiography at admission showed negative $\mathbf{T}$ wave in the precordial and sidewall leads (A). The finding has normalized six months after the prednisolone dose was increased (B).

and is tied to the etiology driving the worsening of the symptoms (2). DCMi, which is a relatively new concept, is diagnosed based on the detection of $>14$ cells $/ \mathrm{mm}^{2}$ infiltrating $\mathrm{T}$ lymphocytes and macrophages in a biopsy specimen $(3,4)$. Autoimmune disease and/or viral infection are considered to be the main causes of infiltrating inflammatory cells in the myocardium; notably, $46 \%$ of DCM cases fulfil the criteria of DCMi (5). A recent prospective randomized trial showed that immunosuppressive therapy was effective for improving the LV systolic function in patients with DCMi (6).

We described the case of a patient with DCM with EGPA who showed myocardial inflammation. Based on the findings, we treated the patient with immunosuppressive therapy. Although non-invasive examinations - including myocardial imaging - did not show any inflammation in the patient's heart, a myocardial biopsy demonstrated striking inflammation in the myocardium that fulfilled the criteria for DCMi. In cases of profound eosinophilia $\left(>5,000\right.$ cells $\left./ \mathrm{mm}^{2}\right)$, eosinophils can have deleterious effects on tissue, particularly in the heart (7). The level of eosinophilia in this case was 3,542 eosinophils/ $\mu \mathrm{L}\left(<5,000\right.$ cells $\left./ \mathrm{mm}^{2}\right)$, which did not lead us to diagnose inflammatory myocarditis.

Systemic inflammatory diseases, such as EGPA and collagen diseases, are known to provoke cardiac dysfunction. Such patients are uniformly treated based on the therapeutic strategies for DCM (e.g., $\beta$-blockers and angiotensinconverting enzyme inhibitors) without trying to assess the myocardial inflammation. However, if uniformly treated, the long-term prognosis of DCMi in patients who receive stan- dard treatment is quite poor in comparison to the prognosis of non-DCMi (5), whereas immunosuppressive therapy has been reported to improve the LV systolic function in patients with DCMi (6). Furthermore, eosinophil-related myocardial injury is reported to progress from the acute necrosis stage (eosinophilic myocarditis) to the scarring (fibrotic) stage (7), suggesting that any delays in the initiation of appropriate therapy could lead to irreversible myocardial damage and LV systolic dysfunction. Accordingly, it is crucial to discriminate between DCMi and non-DCMi, especially in patients suffering from inflammatory disease, such as the patient in the present case.

Myocardial inflammation should be evaluated by biopsy and immunostaining because no other clinical examinations, including ${ }^{67} \mathrm{Ga}$ scintigraphy and cardiac magnetic resonance imaging, identified the patient's myocardial inflammation in the present case. Although high blood levels eosinophils and troponin $\mathrm{T}$ could indicate a worsening of EGPA, which might occur in association with LV systolic dysfunction, the therapeutic approach in this particular case was based on the myocardial biopsy findings.

Autoimmune diseases other than EGPA may also be associated with LV systolic dysfunction caused by the infiltration of inflammatory cells into the myocardium. The addition of immunosuppressive therapy to the standard therapy for DCMi can improve the associated LV systolic dysfunction. In this context, we consider it vitally important to conduct a myocardial biopsy in order to ensure that DCMi patients receive appropriate therapy. 
The authors state that they have no Conflict of Interest (COI).

\section{References}

1. Comarmond C, Pagnoux C, Khellaf M, et al.; French Vasculitis Study G. Eosinophilic granulomatosis with polyangiitis (ChurgStrauss): clinical characteristics and long-term followup of the 383 patients enrolled in the French Vasculitis Study Group cohort. Arthritis Rheum 65: 270-281, 2013.

2. Felker GM, Thompson RE, Hare JM, et al. Underlying causes and long-term survival in patients with initially unexplained cardiomyopathy. N Engl J Med 342: 1077-1084, 2000.

3. Maisch B, Richter A, Sandmoller A, Portig I, Pankuweit S; BMBF-Heart Failure Network. Inflammatory dilated cardiomyopathy (DCMI). Herz30: 535-544, 2005.

4. Caforio AL, Pankuweit S, Arbustini E, et al.; European Society of Cardiology Working Group on M and Pericardial D. Current state of knowledge on aetiology, diagnosis, management, and therapy of myocarditis: a position statement of the European Society of Cardiology Working Group on Myocardial and Pericardial Diseases. Eur Heart J 34: 2636-2648, 2648a-2648d, 2013.

5. Nakayama T, Sugano Y, Yokokawa T, et al. Clinical impact of the presence of macrophages in endomyocardial biopsies of patients with dilated cardiomyopathy. Eur J Heart Fail 19: 490-498, 2017.

6. Frustaci A, Russo MA, Chimenti C. Randomized study on the efficacy of immunosuppressive therapy in patients with virusnegative inflammatory cardiomyopathy: the TIMIC study. Eur Heart J 30: 1995-2002, 2009.

7. Seguela PE, Iriart X, Acar P, Montaudon M, Roudaut R, Thambo JB. Eosinophilic cardiac disease: Molecular, clinical and imaging aspects. Arch Cardiovasc Dis 108: 258-268, 2015.

The Internal Medicine is an Open Access article distributed under the Creative Commons Attribution-NonCommercial-NoDerivatives 4.0 International License. To view the details of this license, please visit (https://creativecommons.org/licenses/ by-nc-nd/4.0/).

(C) 2018 The Japanese Society of Internal Medicine Intern Med 57: 2675-2679, 2018 